\title{
Clinical and Biochemical Evaluation of HIV-Related Lipodystrophy in an Ambulatory Population from the Hospital Universitário Cassiano Antonio de Morais, Vitória, ES, Brazil
}

\author{
Bruno Zanotelli Monnerat, Crispim Cerutti Junior, Sheila Cristina Caniçali and Tânia Reuter Motta \\ Infectious Diseases Division. Hospital Universitário Cassiano Antonio de Morais (HUCAM)
}

\begin{abstract}
We designed a retrospective cohort study to identify factors associated with HIV-1 related lipodystrophy at a tertiary HIV-care center in Vitória, ES, Brazil. Inclusion criteria were documented HIV diagnosis, anti-retroviral therapy and age above 17 years. Highly active antiretroviral therapy (HAART) was initially the exposure variable, but a second analysis was also performed, as a nested case-control, based on the presence or absence of lipodystrophy. Use of protease inhibitors (PI) was associated with an increase in serum triglycerides $(243.7 \pm 189 \mathrm{mg} / \mathrm{dL}$ vs. $172.7 \pm 131$ $\mathrm{mg} / \mathrm{dL}, \mathrm{p}=\mathbf{0 . 0 1 5}$ ), but not of total cholesterol (TC) or HDL fraction levels. Non-nucleoside reverse transcriptase inhibitors (NNRTI) were associated with an increase in serum TC $(180.6 \pm 46.8 \mathrm{mg} / \mathrm{dL}$ versus $162.4 \pm 41.4 \mathrm{mg} / \mathrm{dL} ; \mathrm{p}=$ 0.018 ) and an increase in HDL cholesterol (47.3 $\pm 13.8 \mathrm{mg} / \mathrm{dL}$ versus $23.3 \pm 24.3 \mathrm{mg} / \mathrm{dL} ; \mathrm{p}<0.001$ ), with no significant effect on triglyceride levels. Lipodystrophy was diagnosed in $59.3 \%$ of the patients, but exposure to PI versus NNRTI did not affect the frequency of this disorder $(43.4 \%$ versus $37.2 \% ; p=0.68)$. Serum TC, but not HDL cholesterol or triglyceride levels, was higher among the lipodystrophy cases $(183.8 \pm 47.5 \mathrm{mg} / \mathrm{dL}$ versus $162.1 \pm 35.7 ; \mathrm{p}=0.006)$. Among the controls (patients without lipodystrophy), HDL cholesterol $(45.3 \pm 14.4 \mathrm{mg} / \mathrm{dL}$ vs. $27.1 \pm 26.3$; $\mathrm{p}=0.001)$ and triglyceride levels (178.3 $\pm 146.3 \mathrm{mg} / \mathrm{dL}$ vs. $126.3 \pm 126.9$; $\mathrm{p}=\mathbf{0 . 0 1 3}$ ) also increased, but not TC. In conclusion, lipodystrophy was highly prevalent in our series. Lipid disorders were also frequent and apparently were related to lipodystrophy, except for triglyceride levels.
\end{abstract}

Key-Words: Lipodystrophy, dyslipidemia, Brazil, AIDS, ARV therapy, cohort study.

The lipodystrophies are a heterogeneous group of disorders of the adipose tissue that are characterized by selective redistribution of body fat [1-4]. Highly active antiretroviral therapy (HAART) for HIV has been associated with the development of lipodystrophy in increasing numbers of individuals [2-6], especially after 12 months of therapy [2,7]. This deforming and potentially stigmatizing condition can reduce the adherence and efficacy of anti-HIV therapy [8], and it must not be overlooked by the physicians who care for these patients.

HIV-related lipodistrophy was first described in 1998 [9,10], following a Federal Drug Administration (FDA) warning that anti-retrovirals were associated with hyperglycemia. It is characterized by dorsocervical fat accumulation ("buffalo hump”), central adiposity, peripheral fat wasting and associated laboratory test abnormalities, such as elevated cholesterol and triglycerides, and rarely, type-2 diabetes mellitus [11]. Also, an increase in local vascular distribution and muscle hypertrophy may follow in the fat-wasting regions [2]. The pathophysiological mechanisms that are involved are still not well understood [12,13].

The agents associated with HIV-related lipodystrophy are mainly to protease inhibitors and nucleoside analogue reversetranscriptase inhibitors [2]. As access to HAART is increasing in the developing world, including in Brazil, the need to study Received on 22 January 2008; revised 26 July 2008.

Address for correspondence: Dr. Crispim Cerutti Junior. Departamento de Medicina Social do Centro de Ciências da Saúde da Universidade Federal do Espírito Santo. Av. Marechal Campos, 1468, Maruípe, Vitória, ES, Brazil. Zip code: 29.140-091. Phone: 27-3335-7225. Fax: 273335-7218. E-mail: fil.cris@terra.com.br.

The Brazilian Journal of Infectious Diseases 2008;12(4):264-268. (C) 2008 by The Brazilian Journal of Infectious Diseases and Contexto Publishing. All rights reserved. this condition in such populations is evident. Given these many considerations and concerns about lipodystrophy, we undertook a retrospective cohort study focusing on clinical questionnaires and biochemical markers of dyslipidemia and hyperglycemia to identify factors associated with this condition among HIV-positive patients in Brazil.

\section{Material and Methods}

Setting

We conducted a retrospective cohort study at a tertiary HIV-care center (Hospital Universitário Cassiano Antonio de Morais) in Vitória, ES, Brazil. All patients were taking stateprovided drugs, and laboratory results were provided by the university hospital laboratory (cholesterol levels, triglycerides, glucose) and by LACEN - State Central Laboratory (viral load and CD4 cell count). The research protocol was reviewed and approved by the Ethics on Research Committee of the Centro de Ciências da Saúde da Universidade Federal do Espírito Santo, and all the patients provided written informed consent.

Patients

Members of the study group (BZM, SCC) interviewed 102 HIV-positive patients in the waiting room of the HIV clinic between July 2004 and May 2005. Patients infected with HIV, who were older than 17 years, and who were using any kind of anti-retroviral drug were included in our study. We excluded anyone who had a history of chronic use of corticosteroids, appetite suppressors or stimulants, anabolic steroid therapy or drugs for diabetes control.

\section{Assessment}

After a brief explanation, we conducted a face-to-face interview with each of the subjects meeting the above criteria. 
We utilized the LDCD (Lipodystrophy Case Definition) questionnaire [1], in which patients could report any abnormality concerning fat distribution in the face, neck, arms, legs, buttocks and abdomen subsequent to the diagnosis of HIV infection, graded as none (not noticed), mild (noticeable only if specifically looked for), moderate (easily noted by patient or physician) or severe (obvious to the casual observer) $[1,5]$. We also recorded if the patient had experienced abnormal subdermal fat pads (lipomatosis).

After this initial interview, the patients' weight, height and arterial blood pressure were measured and they were attended normally; their physicians also received the same questionnaire to respond, with the same parameters and grading system. The physicians were not aware of the patient's answers to the questionnaire during the consultations.

The criteria to diagnose lipodistrophy included at least one moderate or severe feature of lipoatrophy, of diffuse fat accumulation, or of lipomatosis that appeared after the diagnosis of HIV infection. Observations of moderate or severe involvement had to be independently confirmed on physical examination by the physician. There was no requirement for such concordance at more than one site.

The researchers then analyzed patient charts for metabolic blood indices, demographic details and HIV infection history (previous CD4 cell count and viral load). Specifically, the results were compared between the initial evaluation at the HIV clinic (we wanted to know the clinical and biochemical profile of our patients at the time of HIV diagnosis) and during the most recent consultation (the profile after the patient had been on anti-retroviral drugs). Whenever this data was not available for those dates, data from the nearest dates was retrieved. The same parameters were evaluated at both time points.

Subjects were allocated into two groups according to the drug class they were using at the time of the interview, namely protease inhibitors (PI) or non-nucleoside analogue reverse transcriptase inhibitors (NNRTI). If the patient was taking both drug classes, he/she was excluded from further analysis. The treatment regimens of both groups also included two different nucleoside analogue reverse transcriptase inhibitors (NRTI). A second analysis was performed comparing the patients based on whether they had lipodystrophy, in a nested casecontrol approach.

\section{Statistical Analysis}

Univariate analysis of potentially-related factors was performed as an initial approach. To evaluate associations between the cases and controls, chi-square tests were performed for each categorical variable, and the "Student" $t$ test was used for each continuous variable. Data were analyzed using SPSS 12.0.1 (Statistical Package for the Social Sciences - Chicago, IL).

\section{Results}

From July 2004 to May 2005, 102 patients were included in the study. Six patients were excluded because they were taking other medications that could affect the study variables (corticosteroids, appetite suppressors or stimulants, anabolic steroid therapy or drugs for diabetes control). Two patients taking neither PI nor NNRTI and 10 patients taking PI and NNRTI concomitantly were also excluded from further analysis. As a consequence, 84 patients remained in the study, 47 in the PI group and 37 in the NNRTI group (Table 1). No significant differences were observed between the demographic variables recorded for these two groups (Table 2).

Treatment with PI was associated with an increase in triglyceride levels when data obtained at the time of the interview (denominated "final") were compared with those retrospectively obtained at the beginning of clinical followup (denominated "initial") (initial $=172.7 \pm 131 \mathrm{mg} / \mathrm{dL}$; final = $243.7 \pm 189 \mathrm{mg} / \mathrm{dL} ; \mathrm{p}=0.015)$. There was no significant difference between initial and final total cholesterol or HDL cholesterol levels in this group of patients (data not shown).

Treatment with NNRTI was associated with an increase in total cholesterol (initial $=162.4 \pm 41.4 \mathrm{mg} / \mathrm{dL}$; final $=180.6 \pm$ $46.8 \mathrm{mg} / \mathrm{dL} ; \mathrm{p}=0.018$ ). A significant increase was also found between initial and final HDL cholesterol levels (initial $=23.3 \pm$ $24.3 \mathrm{mg} / \mathrm{dL}$; final $=47.3 \pm 13.8 \mathrm{mg} / \mathrm{dL} ; \mathrm{p}<0.001)$. There was no significant difference between final and initial triglyceride levels in this group of patients (data not shown). The overall frequency of lipodystrophy was $59.3 \%$ among the subjects, and there was not a significant difference between the two treatment regimens (Table 3 ).

In the nested case-control approach, we included all the eligible patients, regardless of whether they were taking PI, NNRTI, both or none of them. As a consequence, 96 patients were considered for analysis, 57 classified as cases (with lipodystrophy) and 39 as controls (without lipodystrophy). There were no significant differences in the demographic variables when the patients were distributed based on their outcome (Table 4).

There was an increase in total cholesterol (initial: $162.1 \pm$ $35.7 \mathrm{mg} / \mathrm{dL}$; final: $183.8 \pm 47.5 \mathrm{mg} / \mathrm{dL}$; $\mathrm{p}=0.006$ ), but not in HDL cholesterol or triglycerides. Among the controls, HDL cholesterol (initial: $27.1 \pm 26.3$; final: $45.3 \pm 14.4 ; \mathrm{p}=0.001$ ) and triglyceride levels (initial: $126.3 \pm 126.9$; final: $178.3 \pm 146.3$; $p=$ 0.013 ) were elevated, but not total cholesterol (Table 5).

In a separate analysis, we found no significant association between indinavir or estavudine and the upsurge of lipodystrophy (four had lipodystrophy out of 13 patients taking Indinavir; chi-square: $\mathrm{p}=0.44$; odds ratio $=1.64 ; 95 \%$ CI for OR: 0.47 to 5.76) (: 14 had lipodystrophy out of 40 patients taking estavudine; chi-square: $\mathrm{p}=0.34$; odds ratio $=$ 1.5; 95\% CI for OR: 0.65 to 3.46).

\section{Discussion}

To our knowledge, ours is the first study that evaluates the prevalence of both lipodystrophy and dyslipidemia in HIVinfected patients in Brazil. Previous studies have evaluated only the prevalence of lipodystrophy and the efficacy of exercise as an approach to manage this condition [14,15a,15b]. Additionally, four studies dealt specifically with the subject

www.bjid.com.br 
Table 1. Number of HIV-positive patients taking protease inhibitors (PI) or non-nucleoside analogue reverse transcriptase inhibitors (NNRTI) in the outpatient clinic of the Hospital Universitário Cassiano Antonio de Morais from July 2004 to May 2005, after exclusion of those who were using medications that could interfere with lipid metabolism (corticosteroids, appetite suppressors or stimulants, anabolic steroid therapy or drugs for diabetes control) (total: 94 patients).

\begin{tabular}{lc}
\hline Protease inhibitor & Number of patients \\
\hline Ritonavir & 4 \\
Indinavir & 9 \\
Nelfinavir & 21 \\
Lopinavir + Ritonavir & 16 \\
Indinavir + Ritonavir & 4 \\
Atazanavir & 2 \\
Atazanavir + Ritonavir & 1 \\
Total* & 57 \\
\hline Non-nucleoside analogue reverse transcriptase inhibitor & Number of patients \\
\hline Efavirenz & 36 \\
Nevirapine & 11 \\
Total* & 47 \\
*Total is in excess of 10 patients in each group, because there were patients taking both PI and NNRTI.
\end{tabular}

Table 2. Demographic variables observed in HIV-infected patients categorized by the third drug of their therapeutic regimen.

\begin{tabular}{|c|c|c|c|}
\hline Variable & PI & NNRTI & p-value \\
\hline \multicolumn{4}{|l|}{ Age (years) } \\
\hline Mean & 39.8 & 38.5 & 0.555 \\
\hline $95 \% \mathrm{CI}$ & $37.3-42.3$ & $35.5-41.2$ & \\
\hline \multicolumn{4}{|l|}{ Sex } \\
\hline Male & $28(59.6 \%)$ & $23(62.2 \%)$ & 0.809 \\
\hline Female & $19(40.4 \%)$ & $14(37.8 \%)$ & \\
\hline \multicolumn{4}{|l|}{ Treatment duration } \\
\hline Mean (months) & 54.4 & 43.2 & 0.187 \\
\hline $95 \% \mathrm{CI}$ & $43.5-65.4$ & $32.6-53.8$ & \\
\hline \multicolumn{4}{|c|}{ Time since HIV diagnosis } \\
\hline Mean (years) & 6 & 5.35 & 0.380 \\
\hline $95 \%$ CI & $5-6.9$ & $4.32-6.38$ & \\
\hline \multicolumn{4}{|c|}{ Final CD4 count (cells/mm³) } \\
\hline Mean & 396 & 445 & 0.425 \\
\hline $95 \% \mathrm{CI}$ & $328-464$ & 352-537 & \\
\hline
\end{tabular}

Table 3. Distribution of lipodystrophy in patients using protease inhibitors (PI) or Non-nucleoside Analogue Reverse Transcriptase Inhibitors (NNRTI).

\begin{tabular}{|c|c|c|c|c|c|}
\hline \multirow[t]{2}{*}{ Lipodystrophy } & \multicolumn{2}{|c|}{ PI } & \multicolumn{2}{|c|}{ NNRTI } & \multirow{2}{*}{$\begin{array}{c}\text { p-value } \\
\left(\chi^{2}\right) \\
0.641 \\
\end{array}$} \\
\hline & $\mathrm{N}$ & $\%$ & $\mathrm{~N}$ & $\%$ & \\
\hline Yes & 20 & 42.6 & 13 & 35.1 & \\
\hline No & 27 & 57.4 & 24 & 64.9 & \\
\hline
\end{tabular}

of dyslipidemia [16-19]. Our findings were similar to reports from developed countries and to those from studies made in India and Southeast Asia [1,5,6,20,21]. This condition was recognized only relatively recently, and knowledge about the mechanisms and factors involved is still incomplete. Correct treatment is not yet defined. It is not yet clear if lipodystrophy and dyslipidemia are caused by anti-retroviral drugs or if they are a natural consequence of the HIV-infection process $[22,23]$. It has been suggested that there is a complex underlying mechanism, involving both anti-retroviral drugs and particularities of the compromised individual and the environment $[24,25]$. 
Table 4. Demographic variables observed in patients with and without lipodystrophy.

\begin{tabular}{lccc}
\hline Lipodystrophy & Present & Absent & p-value \\
\hline Age (years) & $40 \pm 9$ & & \\
$\quad$ Mean & $38.9-45.5$ & $40 \pm 10$ & 0.861 \\
$\quad$ 95\% CI & $36(63.2 \%)$ & $37.9-45.2$ & \\
Sex & $21(36.8 \%)$ & $19(48.7 \%)$ & 0.160 \\
$\quad$ Male & & $20(51.3 \%)$ & \\
$\quad$ Female & $48 \pm 27$ & $55 \pm 31$ & 0.114 \\
Treatment duration & $37-56$ & $42-67$ & \\
$\quad$ Mean (months) & & & 0.385 \\
$\quad$ 95\% CI & $212 \pm 158$ & $267 \pm 179$ & \\
Initial CD4(cells/mm $\left.{ }^{3}\right)$ & $139-273$ & $219-345$ & \\
$\quad$ Mean & & & \\
$\quad$ 95\% CI & &
\end{tabular}

Table 5. Lipodystrophy state and lipid profile.

\begin{tabular}{lccc}
\hline & Initial & Final & p-value \\
\hline Lipodystrophy present & & & \\
TC & $162.1 \pm 35.7$ & $183.8 \pm 47.5$ & 0.006 \\
HDL & $40.8 \pm 49.8$ & $45.1 \pm 13.7$ & 0.664 \\
TG & $158.3 \pm 128.4$ & $216.3 \pm 187.9$ & 0.120 \\
Lipodystrophy absent & & & \\
TC 180.1 \pm 47 & $189.9 \pm 55.3$ & 0.260 & 0.001 \\
HDL & $27.1 \pm 26.3$ & $45.3 \pm 14.4$ & 0.013 \\
TG & $126.3 \pm 126.9$ & $178.3 \pm 146.3$ & \\
\hline TC=total cholesterol; TG=triglycerides. & & &
\end{tabular}

The prevalence of lipodystrophy among our patients was high, as was the frequency of serum lipid abnormalities, a well-recognized risk factor for coronary disease, stroke and peripheral artery disease. In addition, lipodystrophy is a stigmatizing condition in HIV-infected patients, limiting adherence to treatment and, consequently, our ability to control AIDS.

Cross-sectional and prospective studies recently conducted on this subject have dealt with different methods used for the diagnosis of lipodystrophy $[1,23,26]$. We used a clinical score because of its low cost and ease of application. Sophisticated and expensive diagnostic methods, such as computed tomography, magnetic resonance imaging and dual energy X-ray absorption have been validated, but their use in routine practice is generally not viable in developing countries.

The biochemical abnormalities that we found are similar to those previously reported as a result of therapy with PI and NNRTI $[1,2,27]$. We also performed a separate analysis to evaluate if indinavir or estavudine were independently associated with an upsurge of lipodystrophy [27]. We found no significant association between either drug and lipodystrophy in our cohort, but this result may have been influenced by the relatively small number of patients taking these specific drugs in our study. Though we found no significant increase in triglyceride levels in the patients with lipodystrophy, this could have been a consequence of the high variability in triglyceride levels. In future studies, a larger sample size could reveal significant variation in these parameters.

Unlike previous studies, we found no evidence of a significant association between CD4 cell count or HIV viral load (considered as markers of disease severity) and the occurrence of lipodystrophy. We also found no significant association between treatment duration and the frequency of lipodystrophy. The absence of an association of lipodystrophy with CD4 cell count or HIV viral load could be explained by the relative homogeneity of these latter parameters in our sample. The lack of an association with treatment duration is probably a consequence of the small sample size.

The failure to differentiate between lipoatrophy and lipohypertrophy could be a limitation of our study. However, neither of these manifestations seems to specifically influence any of the biochemical indices [6]. In order to increase the accuracy of the diagnostic criteria, we did not include patients who had only an increase in the abdominal adipose tissue, a precaution also taken in previous studies $[1,6,11]$.

We conclude that lipodystrophy is a common condition among HIV-positive patients under HAART in Brazil. More studies with larger sample sizes would be necessary to fully characterize all the factors that may be involved. Early 
diagnosis of lipodystrophy is important in order to develop strategies to ensure patient adherence to the HAART regimen. Additionally, lipodystrophy can be associated with dyslipidemia, a condition that involves increased cardiovascular risk.

\section{Acknowledgements}

We are grateful to the physicians and patients of the Infectious Diseases clinic of HUCAM, whose participation made this study possible.

\section{Sponsorship and Conflicts of Interest}

The investigators did not receive any funds from pharmaceutical companies, government agencies or universities. The investigators have no competing interests to declare.

\section{References}

1. Carr A., Samaras K., Thorisdottir A., et al. Diagnosis, prediction, and natural course of HIV-1 protease-inhibitor-associated lipodystrophy, hyperlipidaemia, and Diabetes mellitus: a cohort study. Lancet 1999;353:2093-9.

2. Chen D., Misra A., Garg A. Lipodystrophy in Human Immunodeficiency Virus-infected patients. J Clin Endocrinol Metab 2002;87:4845-56.

3. Muñoz-Sanz A., Rodriguez-Vidigal F. F., Domingo P. Pathogenesis of lipodistrophy and metabolic syndromes associated with HIV infection. Med Clin (Barc) 2006;127(12):465-74.

4. Wohl D.A., McComsey G., Tebas, P., et al. Current concepts in the diagnosis and management of metabolic complications of HIV infection and its therapy. Clin Infect Dis 2006;43(5):645-53.

5. Lichtenstein K.A., Ward D.J., Moorman A.C., et al. Clinical assessment of HIV-associated lipodystrophy in an ambulatory population. AIDS 2001;15(11):1389-98.

6. Martinez E., Mocroft A., García-Viejo M.A., et al. Risk of lipodystrophy in HIV-1 infected patients treated with protease inhibitors: a prospective cohort study. Lancet 2001;357:592-8.

7. Haugaard S. B. Toxic metabolic syndrome associated with HAART. Expert Opin Drug Metab Toxicol 2006;2(3):429-45.

8. Duran S., Saves M., Spire B., et al. Failure to maintain long-term adherence to highly active antiretroviral therapy: the role of lipodystrophy. AIDS 2001;15:2441-4.

9. Carr A., Samaras K., Burton S., et al. A syndrome of peripheral lipodystrophy, hyperlipidaemia and insulin resistance in patients receiving HIV protease inhibitors. AIDS 1998;12:F51-8.

10. Carr A., Cooper D.A. Images in clinical medicine. Lipodystrophy associated with an HIV-protease inhibitor. N Engl J Med 1998;339:1296.

11. HIV lipodsytrophy case definition study group. An Objective Case Definition of Lipodystrophy in HIV-Infected Adults: a CaseControl Study. Lancet 2003;361:726-35.
12. Balasubramanyam A., Sekhar R.V., Jahoor F., et al. Pathophysiology of dyslipidemia and increased cardiovascular risk in HIV lipodystrophy: a model for systemic steatosis. Curr Opin Lipidol 2004;15:59-67.

13. Capeau J., Caron M., Vigouroux C., et al. Lipodystrophies related to antiretroviral treatment of HIV infection. Med Sci (Paris) 2006;22(5):511-6.

14. Santos C.P., Felipe Y.X., Braga P.E., et al. Self-perception of body changes in persons living with HIV/AIDS: prevalence and associated factors. AIDS 2005; Suppl 4:S14-21.

15. Terry L., Sprinz E., Stein R., et al. Exercise training in HIV-1infected individuals with dyslipidemia and lipodystrophy. Med Sci Sports Exerc 2006;38(3):411-7.

15b. Florindo A. A., de Oliveira Latorre M. do R., Jaime P. C., Segurado A. A. Leisure time physical activity prevents accumulation of central fat in HIV/AIDS subjects on highly active antiretroviral therapy. Int J STD AIDS 2007;18(10):692-6.

16. Sposito A. C., Caramelli B., Sartori A. M., Ramires J.A.F. The lipoprotein profile in HIV infected patients. Braz J Infect Dis 1997; 1:275-83.

17. Caramelli B., Bernoche Cysm., Sartori A.M.C., et al. Hyperlipidemia related to the use of HIV-protease inhibitors: natural history and results of treatment with fenofibrate. Braz J Infect Dis 2001;5:332-8.

18. Albuquerque E.M., de Faria E.C., Oliveira H.C., et al. High frequency of Fredrickson's phenotypes IV and IIb in Brazilians infected by human immunodeficiency virus. BMC Infect Dis 2005;5(1):47.

19. Malavazi I., Abrao E.P., Mikawa A.Y., et al. Abnormalities in apolipoprotein and lipid levels in an HIV-infected Brazilian population under different treatment profiles: the relevance of apolipoprotein E genotypes and immunological status. Clin Chem Lab Med 2004;42(2):525-32.

20. Lichtenstein K.A., Delaney K.M., Armon C., et al. Incidence of and Risk Factors for Lipoatrophy (Abnormal Fat Loss) in Ambulatory HIV-1 Infected Patients. JAIDS 2003;32:48-56.

21. Pujari S.N., Dravid A., Eknath N., et al. Lipodystrophy and dyslipidemia among patients taking first-line, World Health Organization-recommended highly active antiretroviral therapy regimens in Western India. J Acquir Immune Defic Syndr 2005;39:199.

22. Thaker H.K., Snow M.H. HIV Viral Suppression in the era of antiretroviral therapy. Postgrad Med J 2003;79:36-42.

23. Tien P.C., Grunfeld C. What Is HIV-associated lipodystrophy? Defining fat distribution changes in HIV infection. Curr Opin Infect Dis 2004;17:27-32.

24. Carr A. HIV lipodistrophy: risk factors, pathogenesis, diagnosis and management. AIDS 2003;17(Suppl 1):S141-S8.

25. Mondy K., Tebas P. Cardiovascular risks of antiretroviral therapies. Annu Rev Med 2007;58:141-55.

26. Schwenk A., Breuer P., Kremer G., et al. Clinical Assessment of HIV-Associated Lipodystrophy Syndrome: Bioelectrical Impedance Analysis, Anthropometry and Clinical Scores. Clin Nutr 2001;20:243-9.

27. Grinspoon S., Carr A. Cardiovascular Risk and Body-Fat Abnormalities in HIV-Infected Adults. N Engl J Med 2005;352:48-62. 